\title{
Internet-Based Delivery and Deployment of Document Management Systems
}

\author{
Borko Furht, Florida Atlantic University, Boca Raton, Florida \\ Jim Sheen, CyLex Systems, Boca Raton, Florida \\ Zijad Aganovic, CyLex Systems, Boca Raton, Florida
}

\begin{abstract}
In recent years, business on the Internet has exponentially increased. Consequently, the deployment and management of business applications on the Internet is becoming more and more complex, which requires the development of new Internet architectures suitable to efficiently run these business applications. In this paper, we present a new, revolutionary ASP (Application Service Provider) approach implemented by CyLex Systems in deploying document management systems.
\end{abstract}

\section{Introduction}

Electronic Document Management Systems (DMS) are commercial off-the-shelf software products that complement Database Management Systems (DBMS). DBMS typically store structured data and short records, such as name, address, account number, and social security number. Document management systems store, retrieve, and manage unstructured data, such as files, text, spreadsheets, images, sound clips, multimedia data, and compound documents. The benefits of using DMS include:

- Improved productivity

- Improved cash flow

- Improved customer service

- Better access and use of information

The development of DMS is growing rapidly and they are increasingly being used for business critical applications in financial, services, insurance, manufacturing, government, and many other industries.

In this paper we describe CyLex approach in deploying DMS based on the ASP (Application Service Provider) model. We also evaluate this approach with traditional document management systems.

\section{Present Approaches to Document Management Systems}

The traditional approach in document management systems is based on the client/server architecture. Typically, the DMS runs on the corporate server system, while every client runs proprietary software, which provides user interface and access to documents. The link between clients and the server is via a local area network. This approach requires expensive client software for every desktop.

The advancements in the Internet technologies have contributed to the rapid development of the document management systems in two ways:

- Internet technology makes it more economical to deploy DMS by using browsers instead of expensive proprietary client software for every desktop. Many document vendors offer a combined solution with Internet connectivity through a Web gateway.

- As corporations put internal and external documents on their Intranets and Web sites, there is often the lack of processes associated with storing, securing, finding, and changing corporate documentation. In summary, putting documents on the Web is associated with the problems with managing corporate documentation.

Web-based (or Internet-based) approach in DMS deployment is based on the three-tier architecture, shown in Figure 1.

The system consists of clients (tier 1), the document server (tier 2) and databases (tier 3). Clients are connected to the server through the Intranet or the Internet.

The document server implements the document repository and the document management software. 
The DMS software is used for workflow, process automation services, controlling and managing documents, and other processes throughout distributed enterprises.

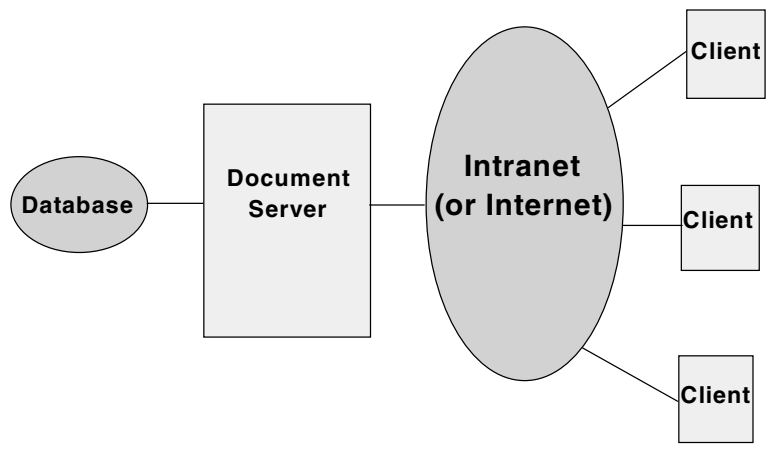

Figure 1. Three-tier architecture for deploying the document management systems.

The document client software typically includes desktop and Intranet (or Internet) clients, which enable users to access and view documents stored in one or more document repositories from their desktops or Web browsers.

These systems are not usually built as XML document managers, however some of the vendors allow that they can be configured to work with XML documents.

In both approaches the DMS software is characterized as corporate-owned in-house application.

\section{The ASP Approach in Software Delivery}

In the last several years a new approach, referred as Application Service Providers, has emerged as a more efficient approach for delivering and maintaining software applications. Application Service Provider is an organization that offers software applications over the Internet. It also coordinates ongoing support, maintenance, and upgrades of applications.

An ASP offers a software application as full service solution, which is revolutionary different from the traditional approaches, where a software application is internally owned and managed solution.

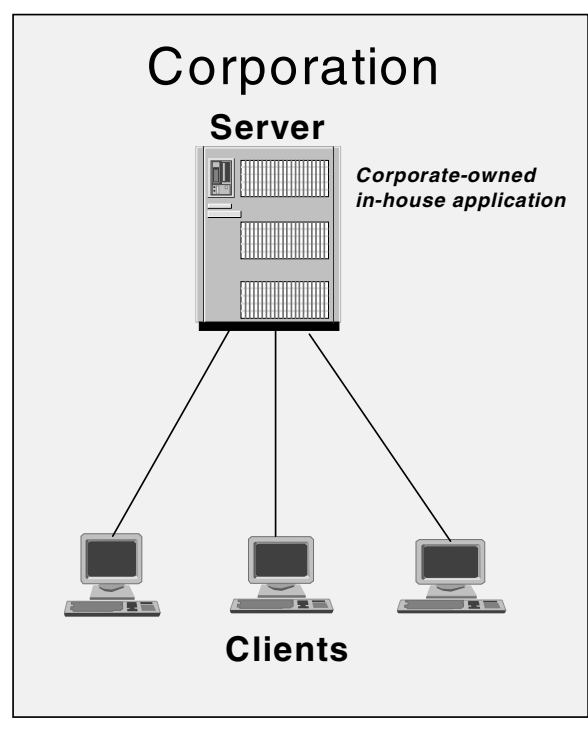

(a) Traditional model

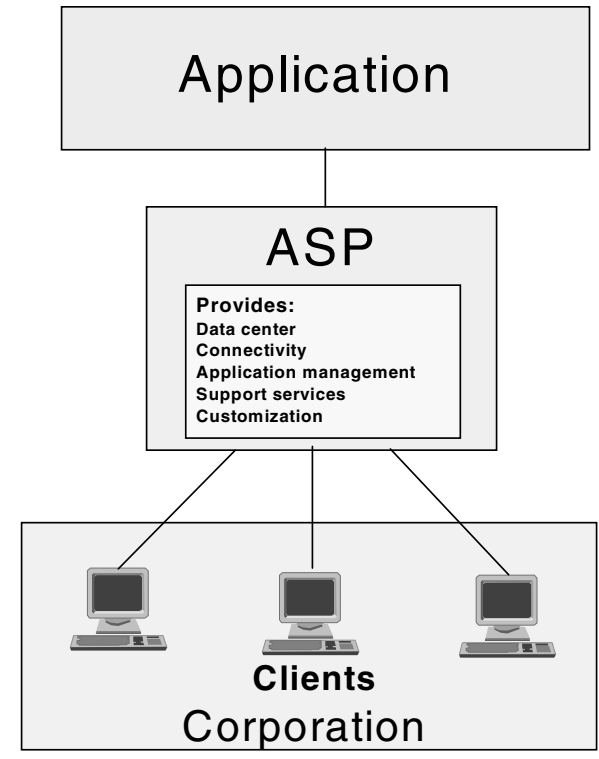

(b) ASP model

Figure 2. Two models in application delivery:

(a) Traditional model based on corporate-owned in-house application, (b) ASP model. 
An ASP rents the use of the software application to customers for a fixed monthly fee. An ASP is typically partnering with:

- Software vendors, which provide software applications

- Application hosting companies that provide data center infrastructure and access to centralized servers, and

- Network service providers that provide connectivity and the network infrastructure.

Figure 2 illustrates the difference between traditional and ASP model in application delivery.

An ASP usually offers from basic services to full and advanced services.

Basic services include basic application maintenance functions, such as sophisticated backups, and application reporting options. It also includes application and data security functions, monitoring networks and servers, and guaranteeing that the application is available.

In addition to basic services, full services include managing application performance and security, and comprehensive customer support and application availability.
In addition to full services, advanced services include application customization and extension services, including customer care, billing, help desk, and others.

The ASP approach provides a number of benefits over the traditional corporate-owned in house applications:

- Superior performance

- Increased security

- Increased reliability

- Increased application scalability

- Reduced total cost of application ownership (TCA) and total cost of IT ownership

In addition, the ASP approach provides customers with significant cost savings. In the traditional application delivery model, besides the software application, the customer must purchase network components, hardware (servers), operating systems, and databases. The company also requires skilled IT professionals to maintain the application. All these expenses are significantly reduced using the ASP approach. With the ASP approach, the company can focus on its core competencies and therefore can shorten deployment timeframes of its products.

\section{CyLex Solution Using the ASP Model}

CyLex Systems is the leading ASP company focusing on delivering document management systems by using the ASP model. CyLex approach and its relationship with the customer (or the client), and independent software vendors is shown in Figure 3.

\section{CyLex Relationships}

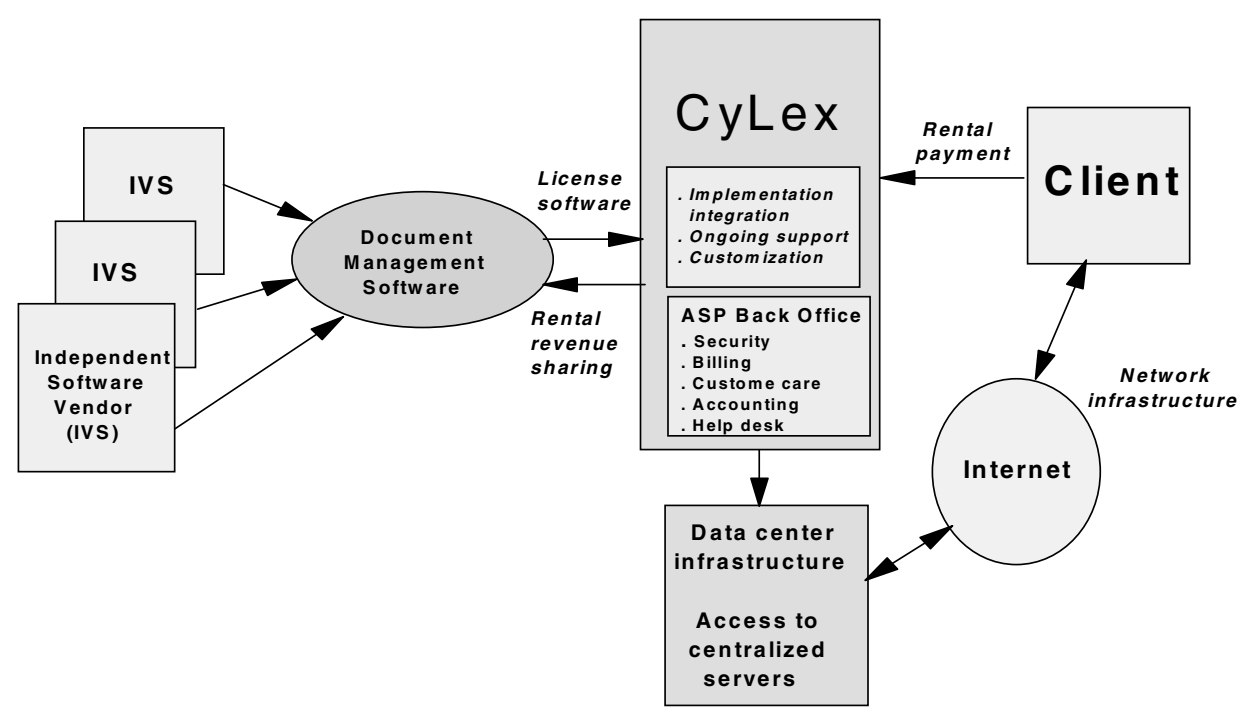

Figure 3. CyLex relationships in the ASP model. 
CyLex is a true ASP company offering advanced services to its customers. Its partners are independent software vendors of DMS applications (such as Documentum) and application hosting companies (such as EMC Inc.). Some DMS companies (such as Feith) use the ASP model to offer their DMS applications. They provide Web access to the DMS software stored on their servers. However, they offer limited services and they are not considered as true ASP companies. For example, they typically do not offer the ASP back office with functions such as security, billing, and customer care.
The CyLex ASP model is based on four-tier architecture, shown in Figure 4. The client is at the first tier, DMS applications comprise the second tier, the ASP back office is at the third tier, and the document databases are at the fourth tier. The first application that CyLex offers as an ASP-enabled DMS is i-DOX, which is built on Documentum's $4 \mathrm{i}$ content management engine. i-DOX is a true ASP-based document management service that offers customers benefit of managing their documents without the need to build, buy, and maintain the hardware, software, and network infrastructure. However, this approach allows offering a variety of DMS applications from different vendors, as shown in Figure 4.

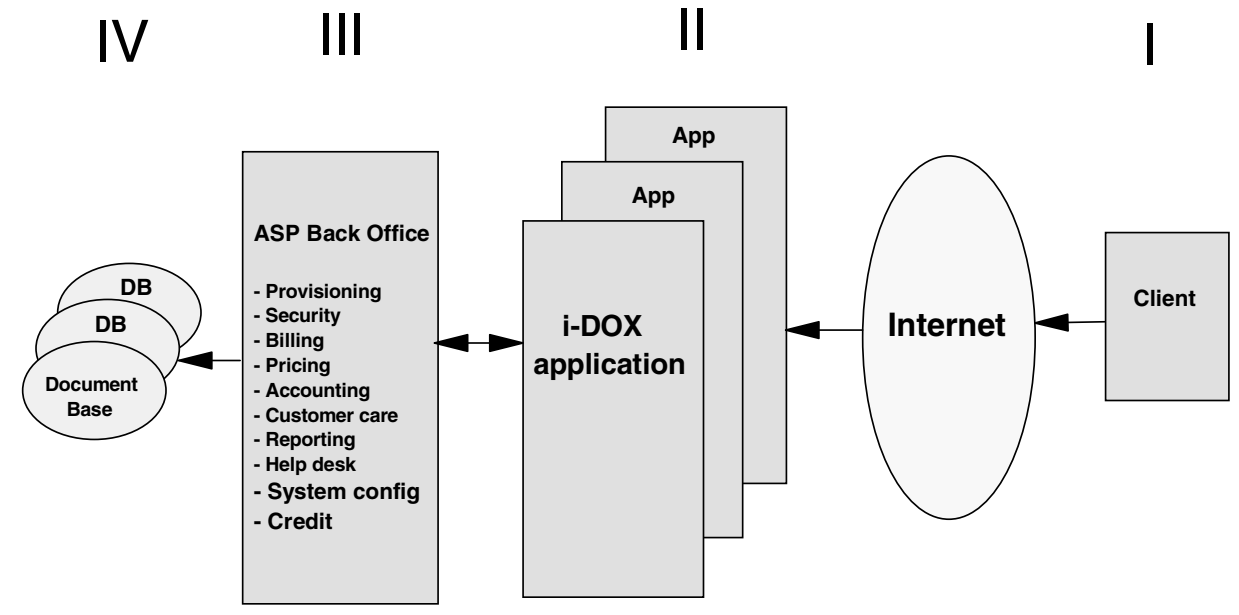

Figure 4. CyLex uses the four-tier architecture to offer ASP-based DMS.

Besides the basic services, which include document management application, i-DOX also provides advanced services including customer care and customer support.

In the four-tier architecture, the customer accesses i-DOX using a PC or workstation running a Web browser. The client software at PC runs user interface including display and updates of documents and supports network transport. The PC is linked to the Web-based application server, which runs the i-DOC application. The application server communicates with a Docbase server that handles document storage and retrieval operations. The Docbase server communicates with an Oracle database server that provides low-level data storage functionality.
The ASP back office provides additional services including provisioning, billing, pricing and packaging, accounting, customer care, reporting, help desk, and system configuration management.

The i-DOX family of service offerings is available in two forms: (i) i-DOX for the small businesses and (ii) iDOX Enterprise Addition for middle-size and large corporations.

i-DOX provides rich, but simple file and retrieval capability that supports TIFF images from scanned documents as well as diverse range of electronic document types. This solution is well suited for 
organizations trying to eliminate the paper documents or to replace microfilm technology. Users can find and access the documents they need via a Web browser from any Internet connected PC.

The i-DOX Enterprise Edition includes all the capabilities of I-DOX plus a rich spectrum of content management functions. Users can manage the life cycle of their information objects through the use of the latest in version control, library services, annotation, and workflow.

Both i-DOX systems support Extensible Markup Language (XML), which means that they can work with XML documents. In order to conduct e-business transactions, companies need a common language for exchanging structured information between their computer systems. HTML, the first generation language of the Internet, is not suited for this task as it defines only the formatting of information, not its meaning. In contrast to HTML, in XML the tags can define the meaning and structure of the information, enabling software applications, such as a DMS, to use that information directly. Therefore, XML has become the second-generation language of the Internet, and it is important that modern document management systems support XML documents.

CyLex software architecture that supports ASP model, shown in Figure 5, consists of five levels.

These five levels are:

- Client level comprises of Netscape and Explorer browsers, document capture software, enablers for legacy applications, and toolbox for custom development.

- Application level contains DMS applications including I-DOX, I-DOX Enterprise Edition, and others.

- Application engine level includes electronic DM, record management, COLD, and others.

- Business management level consists of order entry and provisioning, billing and customer care, on-line help, software license enforcement, and administration and reporting.

- Production system level includes databases, application server farm management, mass storage management, information life cycle, disaster readiness, data output, and operations $\log$ and tracking.

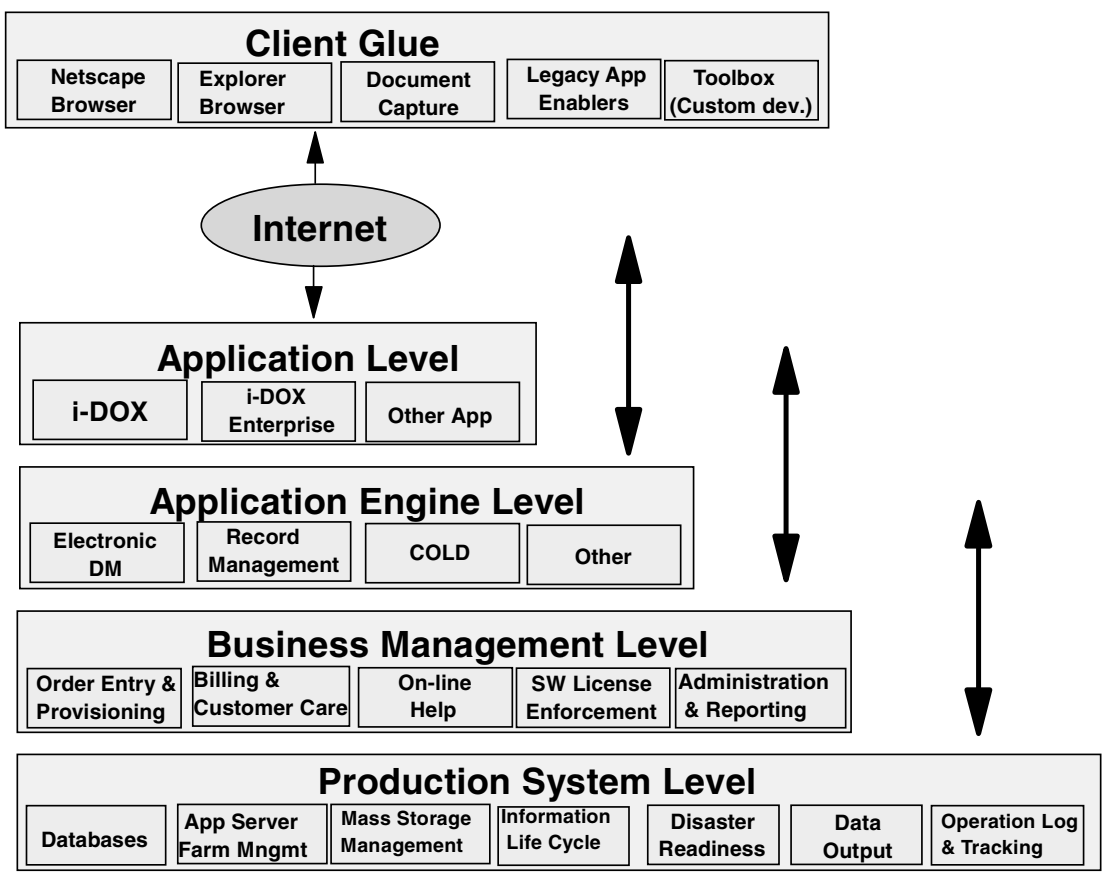

Figure 5. CyLex software architecture. 
Table 1. Evaluation of three approaches for DMS delivery

\begin{tabular}{||l|c|c|c||}
\hline \hline \multicolumn{1}{|c|}{ BENEFITS } & $\begin{array}{l}\text { TRADITIONAL } \\
\text { IN-HOUSE DMS }\end{array}$ & $\begin{array}{c}\text { DMS COMPANY } \\
\text { THAT OFFERS } \\
\text { PRODUCTS } \\
\text { USING ASP } \\
\text { MODEL }\end{array}$ & $\begin{array}{c}\text { CYLEX } \\
\text { APPROACH } \\
\text { TRUE ASP }\end{array}$ \\
\hline Improved productivity & \rfloor & \rfloor & \rfloor \\
\hline $\begin{array}{l}\text { Better access and use } \\
\text { of information }\end{array}$ & \rfloor & \rfloor & \rfloor \\
\hline Financial cost saving & & \rfloor & \rfloor \\
\hline $\begin{array}{l}\text { Focus on core } \\
\text { competencies }\end{array}$ & & & \rfloor \\
\hline Increased security & & & \rfloor \\
\hline Increased scalability & & & \rfloor \\
\hline Customer care & & & \rfloor \\
\hline Extended services & & & \rfloor \\
\hline $\begin{array}{l}\text { Additional DMS } \\
\text { applications }\end{array}$ & & & \\
\hline \hline
\end{tabular}

A contemporary cost analysis should consider the total cost of application ownership (TCA), rather than the total cost associated with specific computing devices. The Tolly Group has developed a model for comparing the TCA of different computing models: (I) traditional desktop, (ii) client-server, and (iii) server-based models. The ASP approach deployed by CyLex uses server-based model, in which applications reside on the servers and can be accessed by users without requiring them to be downloaded to the client. The client can be either thin or fat client.

Tolly Group has analyzed and calculated the total cost of application ownership for a medium-size enterprise of 2,500 users, with 175 mobile users working on the road. Figure 6.

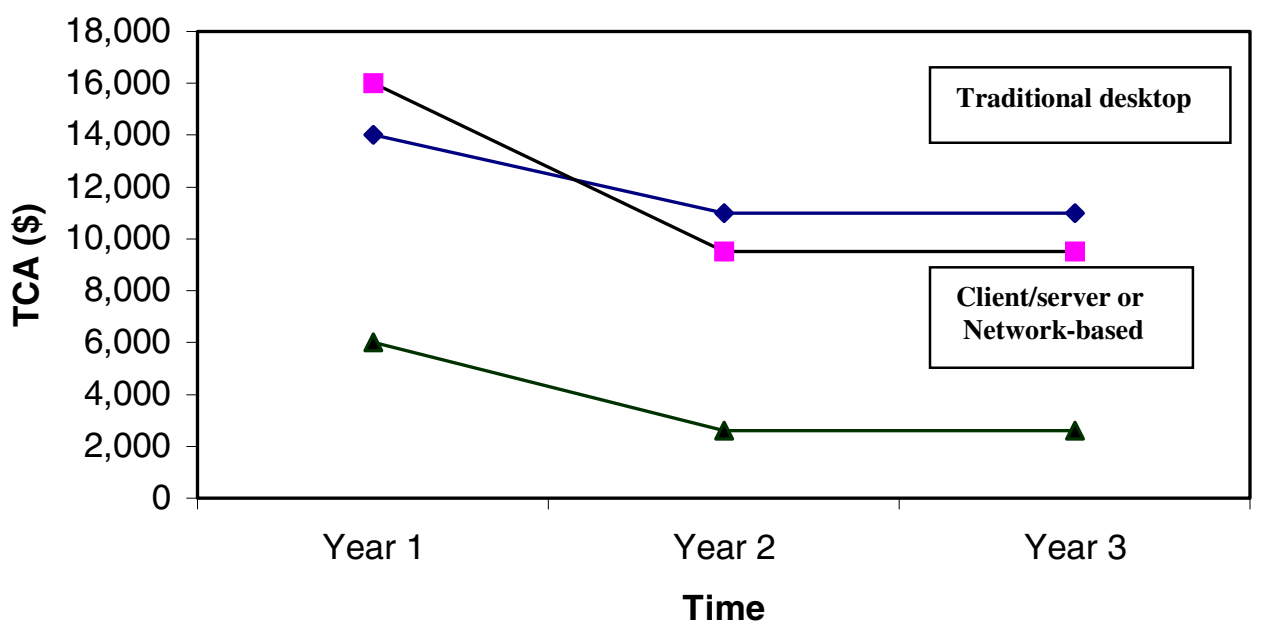

Figure 6. Analysis of total cost of application (TCA) for various computing approaches [2]. 
Traditional desktop computing approach requires relatively high initial cost for hardware, software, network infrastructure, and training $(\$ 14,000)$ as well as very high annual recurring costs for technical support and application maintenance ( $\$ 11,000$ annually).

Client/server and network computing approaches require slightly higher initial investment $(\$ 16,000)$ in order to replace existing client hardware, however annual recurring costs are reduced $(\$ 9,500)$. This model becomes less expensive than the traditional desktop model from the third year forward.

The server-based approach gives the best TCA both in terms of initial costs and annual recurring costs $(\$ 6,000$ and $\$ 2,600$, respectively). The reason for it is that this model allows any type of client to access any application across any type of connection. This model also provides single point for the deployment and management of applications.

\section{Conclusions}

In summary, the server-based model, which is applied in CyLex ASP architecture, is the most efficient and costeffective solution to application deployment and management.

The CyLex revolutionary approach in deploying document management systems is clearly superior to other approaches. The following characteristics make CyLex approach unique and technologically innovative:

- True ASP model has been applied with partners who are the leaders in their areas of expertise, including Documentum (in DMS) and EMC (in application hosting).

- A contemporary four-tier Internet architecture has been implemented.

- The server-based computing, which gives the best TCA, has been used.

- The system offers improved security and scalability.

- The system includes the back office with extended services including billing, customer care, help desk, provisioning, and others.
- The system offers i-DOX as the first DMS application, however the other DMS applications can be integrated into the system.

- End-to-end XML support is provided by i-DOX.

\section{References}

1. P. Dreyfus, "The Second Wave: Netscape on Usability in the Services-Based Internet," IEEE Internet Computing, Vol. 2, No. 2, March/April 1998, pp. 36-40.

2. "Total Cost of Application Ownership," The Tolly Group, Manasquan, NJ, White paper No. 199503, June 1999.

3. J.B. Eichler, R.Y. Roberts, K.W. Evans, and A.L. Carter, "The Internet: Redefining Traditional Business and Giving Rise to New Ones," Report, Stephens Inc., Little Rock, AR, May 1999.

4. "Server-Based Computing," Citrix Systems, white paper, www.citrix.com, 1999.

5. B. Furht, C. Phoenix, J. Yin, and Z. Aganovic, "Internet Architectures for Application Service Providers," Chapter 4 in "Handbook of Internet Computing," Editor-in-Chief B. Furht, CRC Press, Boca Raton, Florida, 2000.

6. G.R. Voth, C. Kindel, and J. Fujioka, "Distributed Application Development for Three-Tier Architectures: Microsoft on Windows DNA," IEEE Internet Computing, Vol. 2, No. 2, March/April 1998, pp. 41-45.

7. C.J. Woodard and S. Dietzen, "Beyond the Distributed Object Decision: Using Components and Java Application Servers as a Platform for Enterprise Information Systems," Distributed Computing, 1998.

8. G. Pour and J. Xu, "Developing 3-Tier Web-Based Enterprise Applications: Integrating CORBA with JavaBeans and Java Servlets," Proc. of the $3^{\text {rd }}$ International Conference on Internet and Multimedia Systems and Applications, Nassau, Bahamas, October 1999.

9. B. Furht, "Application Service Providers - Current Status and Future Trends," International Engineering Consortium, Research Report, 2000. 